\title{
Impact of community gardening on lifestyles' sustainability: quantitative \& qualitative evaluation of a natural experiment
}

\author{
Marion Tharrey ( $\nabla$ mariontharrey@hotmail.fr ) \\ INRA Centre de Montpellier \\ Ashby Sachs \\ University of Colorado Boulder \\ Marlène Perignon \\ INRAE \\ Chantal Simon \\ Laboratoire de Recherche en Cardiovasculaire Metabolisme Diabetologie et Nutrition

\section{Caroline Mejean} \\ INRAE \\ Jill Litt \\ Instituto de Salud Global de Barcelona \\ Nicole Darmon \\ INRAE
}

\section{Research}

Keywords: quasi-experiment, health promotion, food supply, nutrition, physical activity, accelerometer, well-being, connection to nature, urban garden, mental health

Posted Date: July 9th, 2020

DOI: https://doi.org/10.21203/rs.3.rs-39237/v2

License: (a) (1) This work is licensed under a Creative Commons Attribution 4.0 International License. Read Full License 
The authors have withdrawn this preprint from Research Square 